\title{
METODOLOGÍA PARA EVALUAR EL DESGASTE TRIBOLÓGICO DE MAQUINARIA PESADA EN EL SECTOR MINERO
}

\section{METHODOLOGY FOR ASSESSING TRIBOLOGICAL WEAR OF HEAVY MACHINERY IN THE MINING INDUSTRY}

\author{
Brecio Daniel Lazo Baltazar23 y Mario Huatuco Gonzales,
}

Facultad de Ingeniería Mecánica

\section{RESUMEN}

La investigación tuvo por finalidad realizar el estudio de los fenómenos que perjudican el desgaste de los elementos de máquinas a causa del contacto de los elementos en elfuncionamiento de las maquinarias pesadas. La falta de control de: las cargas actuantes, la inclusión de polvo del entorno del cargador, los materiales utilizados, el personal de mantenimiento, perjudican sobre la duración de los sub sistemas que son parte esencial para la producción eficiente de la maquinaria. Hasta el momento, en la mayoría de las empresas han descuidado lo más importante de la producción que es el mantenimiento de los sub sistemas la maquinaria, entonces estas situaciones bajan la producción. La finalidad del mantenimiento es elevar la vida útil de las máquinas reduciendo su impacto ambiental. En el proceso de mantenimiento se generan muchas incertidumbres que influyen en la disponibilidad de las máquinas y tienen efectos negativos en el medio ambiente y otros.

Palabras clave: método, evaluación, tribología, maquinaria minera

\section{ABSTRACT}

The research studied the phenomena that negatively affect the wear of machine elements due to contact while operating heavy equipment. Lack of control: the loads involved, the dust, the materials used, and maintenance personnel damage the duration of the sub systems that are essential for the efficient production of machinery. So far, mostcompanies have neglected the factthat the most important output is the maintenance of the equipment subsystems, which could decrease productivity. The purpose of maintenance is to increase the lifespan of the machines to reduce its environmental impact. During the maintenance process, many uncertainties are generated that affect the availability of machines and impact the environment and others.

Key words: method, evaluation, tribology, mining machinery

\section{INTRODUCCIÓN}

El fin de la tribología será en gran medida el lograr la prolongación de la vida útil de los equipos y componentes mecánicos, a través del control y reducción del desgaste de los mismos. Para ello, se deben estudiar y analizar los diferentes componentes, analizando su desgaste mediante técnicas tribológicas.

La fricción se define como la resistencia al movimiento durante el deslizamiento 0 rodamiento que experimenta un cuerpo sólido al moverse tangencialmente sobre otro con el cual está en contacto. Podemos decir, por lo tanto, que la fricción no es una característica de los materiales, sino del comportamiento del sistema donde estén ubicados esos materiales.

El desgaste es el daño de la superficie y/o eliminación de material de una 0 ambas superficies sólidas en movimiento relativo, ya

\footnotetext{
${ }^{23}$ baltazarlazo@hotmail.com
} 
sea por deslizamiento, rodamiento o impacto. Algunos ejemplos pueden ser: desgaste por abrasión, desgaste por adherencia, corrosión, etc. La lubricación consiste en la interposición y/o aplicación de una capa de un producto que reduzca el coeficiente de fricción entre 2 superficies en rozamiento.

Aplicaciones de la tribología. Son muchos los elementos mecánicos de la industria donde tiene aplicación directa o indirecta la tribología, pero los más habituales son: rodamientos, cojinetes, casquillos, transmisiones, ejes, levas, bielas y elementos de motores térmicos, frenos $y$ embragues de máquinas y vehículos, etc.

\section{MATERIAL Y MÉTODOS}

\section{Material}

El material utilizado para la investigación fue el cargador frontal Volvo L-120 B

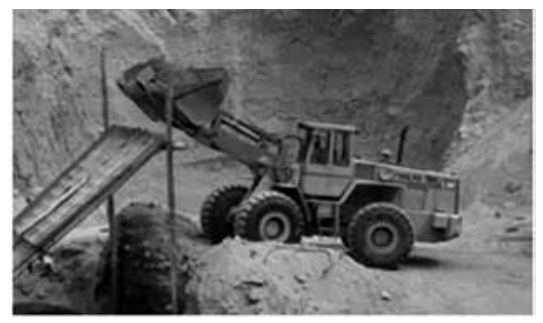

Figura 1: Cargador frontal VOLVO L-120 B

Para identificar el problema se utilizó el estado del arte

Detalles generales

Marca volvo modelo L-120 B, año 1994 fabricación sweden, Motor volvo TD $71 \mathrm{G}$ potencia, 200 hp,Lampón 3,10 m3 llantas nuevas

\section{Sistema hidráulico}

Bomba Princ. I 3050 psi; Bomba Princ. I 196 litros/ min, Bomba Princ. II 2600 psi; Bomba Princ. II 81 litros/min, Bombas regular, Mangueras bueno, Botellas direccionales excelente, Botellas de levante excelente,Pistones direccionales excelente, Pistones de levante excelente .

\section{Métodos}

El tipo de investigación es la descriptiva para ello se utilizó:

Esquema del fenómeno tribológico

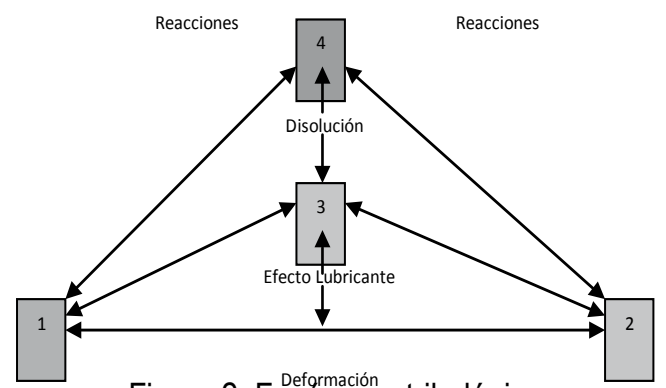

Figura 2: Fenormación tribológico

Revisión de la información bibliográfica con referencia al tema.

Revisión de la tecnología de mantenimiento con efectos de desgaste tribológico

Verificación empírica: utiliza la comprobación de los hechos para formular y resolver problemas.

Entre los métodos empíricos:

Entrevista, observación, análisis de los productos de la actividad, experimento, estudio de caso, historia de vida, etc.

Técnicas de procesamiento y análisis de datos

Para el procesamiento de datos se utilizó la estadística descriptiva e inferencia.

Para el análisis de los datos se utilizó la 
distribución estadísticas temporales dentro del contexto de distribución de Weibull .

\section{RESULTADOS Y DISCUSIÓN}

\section{Tribología}

La Tribología está presente en prácticamente todos los aspectos de la maquinaría, motores y componentes de la industria en general. Los componentes tribológicos más comunes son: rodamientos, embragues, sellos, frenos, anillos de pistones, engranes, cepillos, levas.

Las aplicaciones más comunes de los conocimientos tribológicos, aunque en la práctica no se nombren como tales, son: motores eléctricos y de combustión (componentes y funcionamiento), turbinas, extrusión, rolado, fundición, forja, procesos de corte (herramientas y fluidos), elementos de almacenamiento magnético, prótesis articulares (cuerpo humano).

\section{Tipos de los mecanismos de desgaste}

Es muy importante dejar establecida la necesidad de emplear la máxima rigurosidad en el análisis y diagnóstico de los mecanismos de desgaste. Un diagnóstico errado sobre las causales del deterioro implicará una selección inapropiada del método de recuperación, no lográndose la maximización de vida útil deseada e incluso es posible, mediante una recomendación errónea, acelerar el desgaste en cuestión.

Cuando se pone en marcha piezas mecánicas que experimentan rozamiento, se presentan esquemáticamente tres etapas:

En una primera etapa denominada rodaje, la razón de desgaste es alta, pero disminuye en un breve lapso de tiempo. Este desgaste no compromete el funcionamiento de la pieza; sin embargo, obliga a tomar ciertas precauciones.

Un segundo período llamado marcha normal, representa la vida útil de la máquina. Durante este período el desgaste es débil 0 al menos constante.

Una tercera etapa llamada de envejecimiento, se caracteriza por una razón de desgaste creciente, debido a desgastes cada vez más frecuentes y más graves, hasta deteriorar parcial o totalmente la pieza.

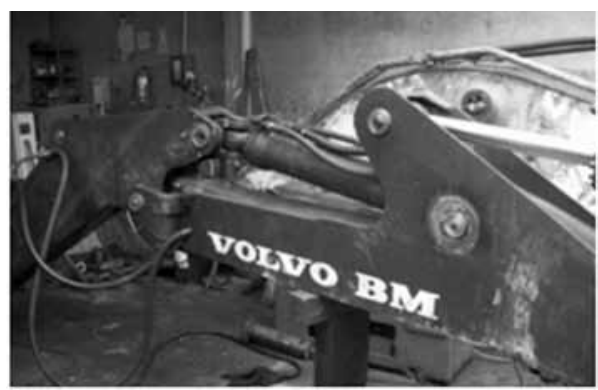

Figura 3: Sistema de movimiento de arena del cargador frontal L-120 B.

\section{Lubricantes antifricción}

La industria de lubricantes constantemente mejora y cambia sus productos a medida que los requerimientos de los automóviles nuevos cambian y nuevos procesos químicos y de destilación son descubiertos. Sabiduría básica de tecnología de lubricación te ayudará a elegir el mejor juego de lubricantes para tu vehículo, 


\section{Prospectiva Universitaria}

sea nuevo o viejo.

Tabla 1: Comparación de viscosidades de lubricantes

\begin{tabular}{|c|c|c|c|c|c|c|c|}
\hline \multicolumn{2}{|c|}{$\begin{array}{l}\text { Kinematic } \\
\text { Viscosities }\end{array}$} & & & & & \multicolumn{2}{|c|}{$\begin{array}{c}\text { Sayboit } \\
\text { Viscosities }\end{array}$} \\
\hline $\begin{array}{l}\mathrm{CSt} \\
\text { (1) } 40^{\circ} \mathrm{C}\end{array}$ & (बide & $\begin{array}{l}\text { Iso } \\
\text { VG }\end{array}$ & $\begin{array}{l}\text { A GuaA } \\
\text { Grade }\end{array}$ & $\begin{array}{l}\text { SAE } \\
\text { Crantease }\end{array}$ & $\begin{array}{l}\text { SAE } \\
\text { Gear }\end{array}$ & $\begin{array}{l}\text { sus } \\
\text { @100" }\end{array}$ & $\begin{array}{l}\text { sus } \\
\text { Q210F }\end{array}$ \\
\hline $2000-$ & $\Gamma^{70}$ & & & & & $\Gamma^{10000}$ & \\
\hline & -60 & & & & & -8000 & - \\
\hline $800-$ & & 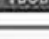 & in & & & -4000 & -200 \\
\hline $600-$ & -40 & 692 & 8 & & 190 & -3000 & - \\
\hline $500-$ & & & & & & & - \\
\hline $400-$ & & 980 & 7 & & 140 & -2000 & - \\
\hline $300-$ & -20 & 820 & 6 & 60 & 110 & -1500 & E \\
\hline $200-$ & & 202 & 5 & 50 & & -1000 & -90 \\
\hline & & 150 & 4 & 40 & 90 & -800 & -80 \\
\hline $100-$ & & & & & $85 \%$ & -500 & -70 \\
\hline$\infty 0-$ & $E_{9}^{10}$ & & & & & -400 & -60 \\
\hline $60-$ & -8 & & 2 & & dow & -300 & -55 \\
\hline $50-$ & -7 & & & 20 & & -200 & ${ }^{-50}$ \\
\hline $40-$ & 6 & 46 & 1 & & & & -45 \\
\hline $30-$ & 5 & 32 & & $15 \%$ & $75 \%$ & $F^{-150}$ & \\
\hline $20-$ & $L^{-4}$ & 22 & & sw ow & & -100 & -40 \\
\hline $10-$ & & 10 & & & & $L_{\infty}$ & \\
\hline
\end{tabular}

Fuente: www.noria.com/sp/recursos/lubetips/ v22/t27.asp

\section{Programa de análisis de aceites}

El análisis de aceite como parte del mantenimiento predictivo, es una herramienta que nos permite determinar el estado de los aceites y el nivel de desgaste de la maquinaria, a través del cual se puede anticipar las fallas, otorgar las soluciones para controlar el progreso de las mismas y aumentar el tiempo de vida de las maquinarias.

Servicio integral de lubricación

Este servicio permite a los clientes el traspaso de sus operaciones de lubricación a una empresa experta en lubricación de maquinaria.
Mediante este servicio el cliente puede aumentar la disponibilidad de los equipos, incremento de la producción, reducción de costos en la lubricación de planta.

El deslizamiento entre superficies sólidas se caracteriza generalmente por un alto coeficiente de fricción y un gran desgaste debido a las propiedades específicas de las superficies. La presencia de una capa de material ajeno en la interfase no puede ser garantizada durante el proceso de deslizamiento, por lo tanto, se aplican lubricantes para disminuir la fricción y el desgaste. El término lubricación se aplica a dos situaciones: la lubricación sólida y la lubricación de película de fluido (líquido o gaseoso). 


\section{Acabado superficial}

Estas imperfecciones deben ser medidas por el departamento de calidad del taller. El diseñador de la pieza debe decidir qué tipo de superficies son aptas para el funcionamiento del conjunto y reflejarlo en los planos.

Dependen del material empleado así como de las condiciones mecánicas como la carga, la velocidad y la frecuencia de contacto. Por ejemplo, cuando dos partes se mueven en relación a otra, están en contacto las superficies, pero sus cuerpos suponen una discontinuidad. Es decir, no solo es la superficie del metal la única que constituye el contacto, sino también una capa de óxido natural, una capa de moléculas orgánicas, una capa de absorbentes químicos y una capa de absorbentes físicos. Además existe un coeficiente de fricción entre las dos partes, cuya fórmula nunca varía en relación a las fuerzas tangenciales de fricción y las fuerzas normales de presión sobre la superficie, ya que este coeficiente de fricción es constante.

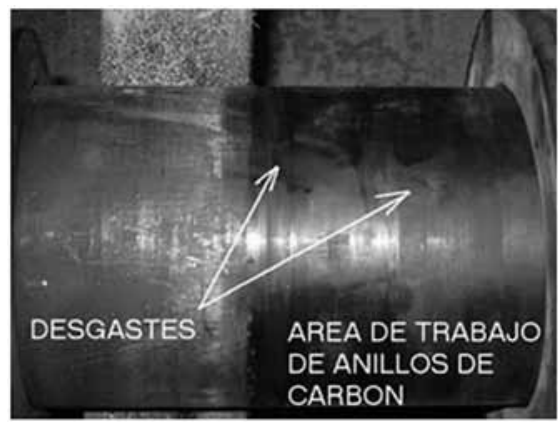

Figura 4: Imperfecciones superficiales

\section{Ajustes $\mathrm{y}$ tolerancias}

Para que un mecanismo funcione correctamente, es necesario que las distintas piezas que lo componen estén acopladas entre sí en condiciones bien determinadas.

Se entiende por ajuste, la relación mecánica existente entre dos piezas cuando se acoplan entre sí (una de ellas encaja en la otra); esta relación resulta con "juego" (holgura) cuando las dos piezas pueden moverse entre sí con cierta facilidad, y con "aprieto" cuando verificado el encaje las piezas han quedado sin posibilidad de movimiento relativo entre ellas.

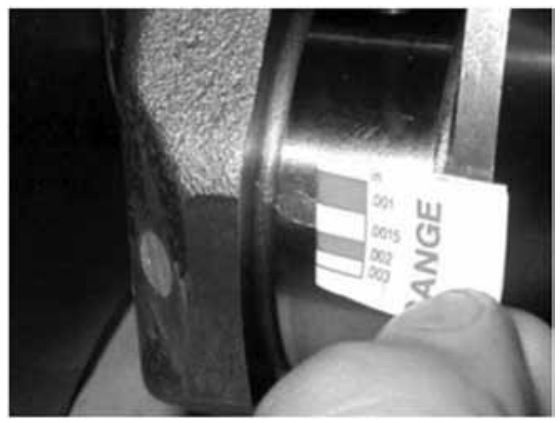

Figura 5: Medición de la tolerancia de ajuste

\section{Tolerancia de forma}

En determinadas ocasiones, como por ejemplo: mecanismos muy precisos, piezas de grandes dimensiones, etc., la especificación de tolerancias dimensionales puede no ser suficiente para asegurar un correcto montaje y funcionamiento de los mecanismos.

Las siguientes figuras muestran tres casos donde una de las piezas puede ser correcta desde el punto de vista dimensional (diámetros de las secciones dentro de tolerancia) y no ser apta para el montaje: en el primer caso tendríamos un defecto de rectitud, en el segundo caso tendríamos un defecto de coaxialidad, y en el tercer caso tendríamos un defecto de perpendicularidad.

Vemos, pues, que en la fabricación se producen irregularidades geométricas que pueden afectar a la forma, posición y orientación de los diferentes elementos constructivos de las piezas. 

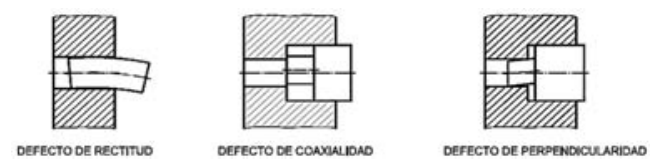

Figura 6: Efectos de mal alineamiento

Una tolerancia dimensional aplicada a una medida ejerce algún grado de control sobre desviaciones geométricas, por ejemplo: la tolerancia dimensional tiene efecto sobre el paralelismo y la planicidad. Sin embargo, en algunas ocasiones la tolerancia de medida no limita suficientemente las desviaciones geométricas; por tanto, en estos casos se deberá especificar expresamente una tolerancia geométrica, teniendo prioridad sobre el control geométrico que ya lleva implícita la tolerancia dimensional.

\section{Materiales usados para reducir el desgaste}

Con los avances tecnológicos, los nuevos sistemas de materiales y procesos se están desarrollando y están creciendo más rápido que nunca. En el pasado, el acero y aluminio fueron más dominantes para el diseño del producto. Esto no es ya el caso. Con la creciente toma de conciencia y las necesidades de los clientes, la ignorancia de oportunidades ofrecidas por avanzados sistemas de materiales tales como los materiales compuestos pueden causar descenso de la competitividad y puede conducir a la pérdida de mercado.

Hay dos principales razones por las cuales conviene que en un ingeniero participe en el proceso de selección del material.

Para rediseñar un producto existente de mejor rendimiento, menor costo, mayor fiabilidad, disminución de peso, etc.

Para seleccionar un material de un nuevo producto 0 aplicación

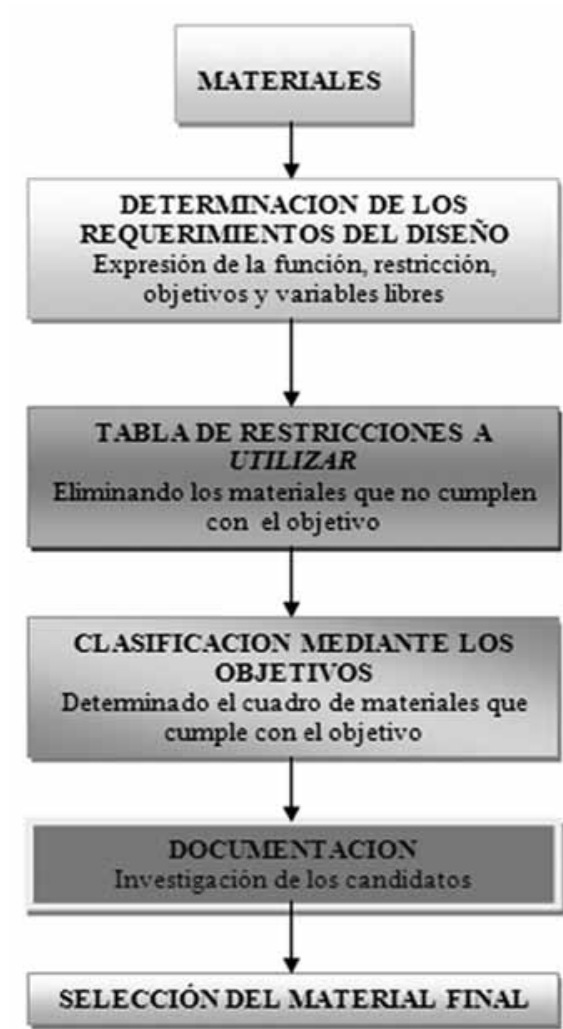

Figura 7: Proceso de selección del material

\section{CONFIABILIDAD}

Confiabilidad es la probabilidad de que un sistema ejecute su función de intención sin fallar para un intervalo específico, bajo condiciones establecidas. Se define como la probabilidad de supervivencia en un determinado tiempo.

$$
R(t)=1-F(t)=1-\int_{0}^{t} f(t) d t=\int_{t}^{\infty} f(t) d t
$$

\section{DISCUSIÓN}

Tribología

La tribología se centra en el estudio de tres 
fenómenos; la fricción entre dos cuerpos en movimiento, el desgaste como efecto natural de este fenómeno y la lubricación como un medio para evitar el desgaste.

Las tareas del especialista en tribología (tribólogo) son las de reducir la fricción y desgaste para conservar y reducir energía, lograr movimientos más rápidos y precisos, incrementar la productividad y reducir el mantenimiento.

\section{Tipos de los mecanismos de desgaste}

El desprendimiento de material en un componente mecánico puede variar notablemente, de muy intenso a despreciable. En cualquier caso, ello puede significar una pérdida de eficiencia y/o la inutilidad completa del sistema.

Es muy importante dejar establecida la necesidad de emplear la máxima rigurosidad en el análisis y diagnóstico de los mecanismos de desgaste. Un diagnóstico errado sobre las causales del deterioro implicará una selección inapropiada del método de recuperación, no lográndose la maximización de vida útil deseada e incluso es posible, mediante una recomendación errónea, acelerar el desgaste en cuestión.

\section{Lubricantes antifricción}

El análisis de aceite como parte del mantenimiento predictivo, es una herramienta que nos permite determinar el estado de los aceites y el nivel de desgaste de la maquinaria, a través del cual se puede anticipar las fallas, otorgar las soluciones para controlar el progreso de las mismas y aumentar el tiempo de vida de las maquinarias.

Las desviaciones analíticas de los análisis anteriores son la clave para determinar los problemas de un equipo.

Un verdadero sistema de análisis:

Es una prueba continua y a intervalos regulares.
Es una herramienta proactiva y no reactiva.

A medida que se generan los historiales, el diagnóstico es cada vez más preciso y efectivo.

El deslizamiento entre superficies sólidas se caracteriza generalmente por un alto coeficiente de fricción y un gran desgaste debido a las propiedades específicas de las superficies. La presencia de una capa de material ajeno en la interfase no puede ser garantizada durante el proceso de deslizamiento, por lo tanto, se aplican lubricantes para disminuir la fricción y el desgaste. El término lubricación se aplica a dos situaciones: la lubricación sólida y la lubricación de película de fluido (líquido o gaseoso).

\section{Acabo superficial}

Mediante las tolerancias dimensionales y geométricas se garantiza la intercambiabilidad de piezas dentro de un conjunto, pero no se garantiza el estado de las superficies de la pieza, factor que influye en el funcionamiento del mecanismo.

Estas imperfecciones deben ser medidas por el departamento de calidad del taller. El diseñador de la pieza debe decidir qué tipo de superficies son aptas para el funcionamiento del conjunto y reflejarlo en los planos.

\section{Ajustes y tolerancias}

Para que un mecanismo funcione correctamente, es necesario que las distintas piezas que lo componen estén acopladas entre sí en condiciones bien determinadas.

\section{Tolerancia de forma}

Una estricta tolerancia de forma asegura, por ejemplo, una gran precisión. La cilindricidad (desviación del diámetro de las superficies de 
fricción de la medida cilíndrica ideal teórica) es de máx. 5/100 mm.

\section{Materiales usados para reducir el desgaste}

Dependen del material empleado así como de las condiciones mecánicas como la carga, la velocidad y la frecuencia de contacto. Por ejemplo, cuando dos partes se mueven en relación a otra, están en contacto las superficies, pero sus cuerpos suponen una discontinuidad. Es decir, no solo es la superficie del metal la única que constituye el contacto, sino también una capa de óxido natural, una capa de moléculas orgánicas, una capa de absorbentes químicos y una capa de absorbentes físicos. Además existe un coeficiente de fricción entre las dos partes, cuya fórmula nunca varía en relación a las fuerzas tangenciales de fricción y las fuerzas normales de presión sobre la superficie, ya que este coeficiente de fricción es constante.

\section{Modelo de confiabilidad}

Una forma más general de 3 parámetros de la Weibull incluye un parámetro de tiempo de espera (localización ó desplazamiento).

Las fórmulas se obtienen reemplazando t por (t口).

No puede ocurrir una falla antes de $\square$ horas, el tiempo comienza en $\square$ no en 0 .

$\operatorname{CDF}: F(t)=1-e^{-\left(\frac{t-\gamma}{\eta}\right)^{\beta}}$

CONFIABILIDAD : $\mathrm{R}(\mathrm{t})=\mathrm{e}^{-\left(\frac{\mathrm{t}-\gamma}{\eta}\right)^{\beta}}$

PDF : $\mathrm{f}(\mathrm{t})=\frac{\beta}{\eta}\left(\frac{\mathrm{t}-\gamma}{\eta}\right)^{\beta-1} \mathrm{e}^{-\left(\frac{\mathrm{t}-\gamma}{\eta}\right)^{\beta}}$

MEDIA $: m=\gamma+\eta \Gamma\left(1+\frac{1}{\beta}\right)$

MEDIANA: $\gamma+\eta(\ln 2)^{1 / \beta}$

VARIANZA: $\eta^{2} \Gamma\left(1+\frac{2}{\beta}\right)-\left[\eta \Gamma\left(1+\frac{1}{\beta}\right)\right]^{2}$

TASADE FALLA: $\mathrm{h}(\mathrm{t})=\frac{\beta}{\eta}\left(\frac{\mathrm{t}-\gamma}{\beta}\right)^{\beta-1}$

Los resultados que se muestran fueron alcanzados por la integración del análisis de la variación de holgura y rugosidad, variación de la forma de la envolvente, desgaste de los engranajes y análisis del lubricante.

Éstas reflejan los efectos en la disminución del caudal y presión de la bomba hidráulica y por ende la confiabilidad.

Tabla 2: Medición del desgaste de los engranajes.

\begin{tabular}{ccccccc}
\hline Muestra & Material & Dureza & Rugosidad mm & Tiempo seg & rpm & Peso gr \\
\hline Blanco & 50CrMo4 & 45 HRC & 0.200 & 0 & 0 & 178.369 \\
1 & 50CrMo4 & 45 HRC & 0.200 & 3600 & 1200 & 178.360 \\
2 & 50CrM04 & 45 HRC & 0.200 & 7200 & 1200 & 178.300 \\
3 & 50CrMo4 & $45 \mathrm{HRC}$ & 0.200 & 10800 & 1200 & 178.250 \\
4 & 50CrMo4 & $45 \mathrm{HRC}$ & 0.200 & 14400 & 1200 & 178.200 \\
5 & 50CrM04 & $45 \mathrm{HRC}$ & 0.201 & 18000 & 1200 & 178.200 \\
6 & 50CrM04 & $45 \mathrm{HRC}$ & 0.201 & 21600 & 1200 & 178.100 \\
7 & 50CrMo4 & 45 HRC & 0.201 & 25200 & 1200 & 178.050 \\
8 & 50CrMo4 & 45 HRC & 0.201 & 28800 & 1200 & 178.050 \\
\hline
\end{tabular}




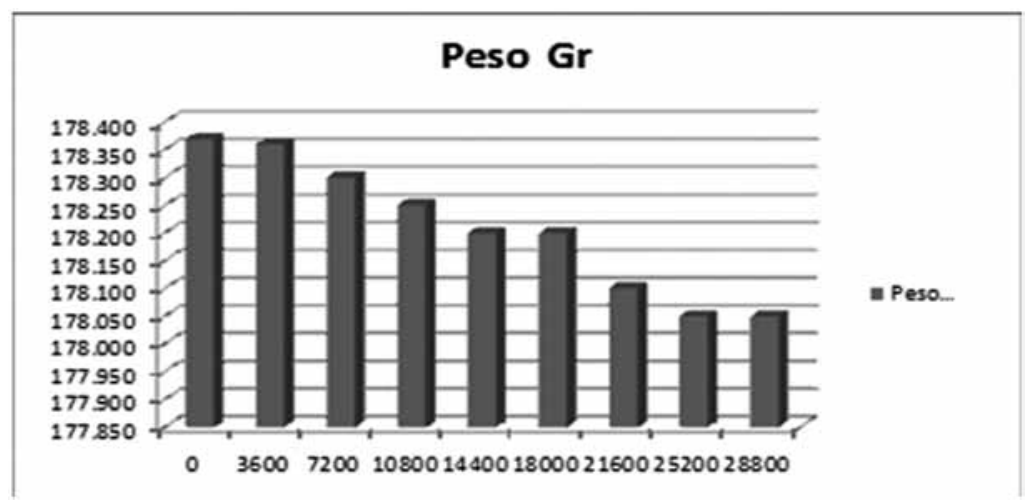

Fuente: Elaboración propia (pruebas, máquina tribológica)

Se observa que es de vital importancia que son parte importante en la longevidad de los las variables (contacto, fricción y lubricación), elementos de la maquinaria pesada.

Tabla 3: Análisis del lubricante

\begin{tabular}{cccccccc}
\hline Muestra & Tiempo seg & $\begin{array}{c}\text { Viscosidad } \\
100^{\circ} \mathbf{C} \text { cSt }\end{array}$ & $\begin{array}{c}\text { No. Acides } \\
\mathbf{m g} \text { KOH/g }\end{array}$ & $\begin{array}{c}\mathrm{H}_{2} \mathbf{O} \% \\
(\mathbf{m} / \mathbf{m})\end{array}$ & $\begin{array}{c}\text { Espumas } \\
\text { cc/seg }\end{array}$ & $\begin{array}{c}\text { Emulsion } \\
\text { min/ml }\end{array}$ & $\begin{array}{c}\mathrm{Fe} \\
\mathbf{p p m}\end{array}$ \\
\hline Blanco & 0 & 42 & 0.2 & 0 & 0 & 0 & 0 \\
1 & 3600 & 42.8 & 0.21 & 0 & 0 & 0 & 0 \\
2 & 7200 & 42.25 & 0.21 & 0 & 0 & 0 & 1 \\
3 & 10800 & 42.35 & 0.21 & 0.05 & 0 & 0.5 & 1 \\
4 & 14400 & 42.38 & 0.22 & 0.05 & 0 & 0.5 & 1 \\
5 & 18000 & 42.38 & 0.22 & 0.05 & 0 & 0.5 & 1.2 \\
6 & 21600 & 42.42 & 0.22 & 0.05 & 0 & 0.5 & 1.2 \\
7 & 25200 & 42.42 & 0.23 & 0.07 & 0 & 0.5 & 1.5 \\
8 & 28800 & 42.39 & 0.23 & 0.07 & 0 & 0.7 & 1.5 \\
\hline
\end{tabular}

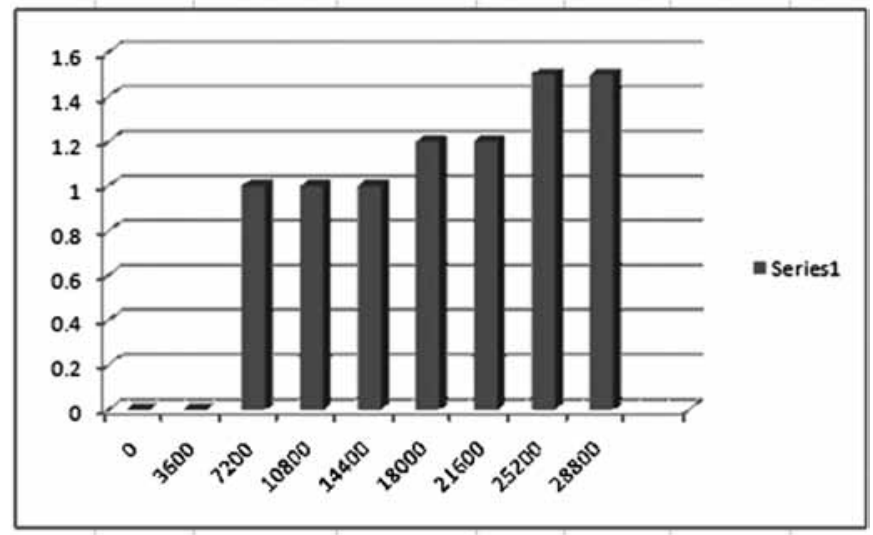

Fuente: elaboración propia (pruebas realizadas, maquina tribología). 
El análisis del lubricante es un indicador que REFERENCIA BIBLIOGRÁFICA sirve para mejorar la planificación y gestión del mantenimiento de las maquinarias pesadas, de manera que se logre cumplir con la longevidad admisible.

La tribología es crucial para la maquinaría moderna que utiliza superficies rodantes $y / 0$ deslizantes.

La aplicación de los conocimientos de la tribología en estas prácticas deriva en:

Ahorro de materias primas

Stolarski.T.A. Tribology in machine design.first published. USA: Oxford Butterworth-Heinemann; 1990.

Williams J.A. Engineering tribology first published. USA: Oxford University Press; 1994.

Niemann Gustav. Elementos de máquinas. Primera edición. Barcelona: Labor, S.A.; 1994.

Bautista Paz, Emilio. Ingeniería de mantenimiento. Primera edición. Perú: PUCP; 2009

Aumento en la vida útil de las herramientas y la maquinaría

Ahorro de recursos naturales

Roberto Hernández Sampieri, Carlos Fernández Collado, Pilar Baptista Lucio. Metodología de la investigación. Tercera edición. México: McGraw Hill; 2005.

Ahorro de energía

Norton Robert L. Machine design and integrated

Protección al medio ambiente

Ahorro económico approach. First Published. E.U.A.: Prentice Hall; 1998.

Shigley, Joseph Edward. Diseño de elementos de máquina. Octava edición. España: McGraw Hill; 2004. 